\title{
Quiste óseo simple: Reporte de un caso y revisión de la literatura
}

\section{Simple bone cyst: a case report and a literature review}

\author{
Chrcanovic BR*, López Alvarenga R**, Freire-Maia B***
}

\section{RESUMEN}

El quiste óseo simple, es una cavidad intraósea sin recubrimiento epitelial, siendo considerado un pseudoquiste. Clínicamente, la lesión suele ser asintomática y descubierta por casualidad en un examen radiológico de rutina. Su etiología y patogenia no están aún definitivamente establecidas. El tratamiento tradicional consiste en realizar una exploración quirúrgica y curetaje de la pared ósea. En este artículo se presenta un caso de quiste óseo simple localizado en la sínfisis mandibular, de dimensión mediana, que acomete a un paciente de sexo masculino de 14 años de edad, revelando datos clínicos, aspectos diagnósticos, imagenológicos, así como su tratamiento.

Palabras clave: Quiste óseo simple, quiste óseo solitario, quiste óseo traumático, cavidad ósea idiopática, diagnóstico diferencial, tratamiento.

\section{SUMMARY}

The simple bony cyst is an intraosseous cavity without epithelial lining, being considered a pseudocyst. Clinically, the lesion is usually asymptomatic and discovered by accident in a routine radiological examination. Its etiology and patogenesis are not yet definitely established. The traditional treatment consists of realizing a surgical exploration and curettage of the bony wall. This article presents a case of simple bony cyst located in the mandibular symphysis, of median dimension of a masculine patient of 14 years of age, describing clinical, diagnostic aspect, imaging, as well as the treatment.

Key words: Simple bone cyst; solitary bone cyst; traumatic bone cyst; idiopathic bone cavity; differential diagnosis, treatment.

Fecha de recepción: 15 de febrero de 2010.

Aceptado para publicación: 18 de marzo de 2010.

* Departamento del Servicio de Cirugía y Traumatología Buco Maxilofacial del Hospital María Amélia Lins (HMAL) de Belo Horizonte. Brasil.

** Alumno del curso de especialización en implantología oral de la Universidad de Santiago de Compostela. España.

*** Prof. Asistente de Cirugía Buco Maxilofacial. Facultad de Odontología da PUC Minas (Pontifícia Universidade Católica). Belo Horizonte. Brasil.

Chrcanovic BR, López Alvarenga R, Freire-Maia B. Quiste óseo simple: reporte de un caso y revisión de la literatura. Av. Odontoestomatol 2011; 27 (4): 207-213. 


\section{INTRODUCCIÓN}

El quiste óseo simple (QOS) es una cavidad intraósea sin recubrimiento epitelial con un contenido seroso y/o sanguinolento que en algunos casos puede estar ausente $(1,2)$.

La incidencia del quiste óseo simple es aproximadamente $1,1 \%$ de los quistes maxilares (3). La lesión es diagnosticada con mayor frecuencia en pacientes que se encuentran durante la segunda década de la vida y no hay una predilección por el género $(4,5)$. La localización más predominante de los QOS es en el hueso mandibular, encontrándose con mayor frecuencia en el cuerpo mandibular y seguido de la región sinfisiaria. Un menor número de casos se reportan en la rama y el cóndilo. Cuando se presenta en el maxilar superior, la zona más afectada es el sector anterior $(2,4)$.

Clínicamente, es casi siempre asintomático y suele descubrirse durante una exploración radiográfica de rutina. En la mayoría de las veces los dientes de la zona presentan vitalidad pulpar (6-8).

En los exámenes radiográficos el QOS usualmente se presenta como un área radiolúcida bien delimitada, con un borde irregular de tamaño variable, sin desplazamiento dentario y sin reabsorción radicular $(6,9)$.

Histológicamente es muy variable. La lesión aparece como una cavidad del hueso medular, que ocasionalmente puede presentar un delgado tejido conjuntivo sin evidencia de un componente epitelial $(3,6)$.

El tratamiento recomendado es la exploración quirúrgica seguida del curetaje de la pared ósea con la finalidad de producir sangrado en la cavidad y la regeneración del tejido óseo $(6,10)$.

El objetivo de este trabajo es presentar un caso de QOS localizado en la región sinfisiaria, de extensión mediana, en el cual se discuten los aspectos característicos del caso y hacer una breve revisión de la literatura.

\section{CASO CLÍNICO}

Paciente de sexo masculino de 14 años de edad, fue remitido a la Clínica de Cirugía Oral de la Facultad de Odontología de la PUC-Minas (Pontifícia Universidade Católica) por su ortodoncista por presentar una imagen radiolúcida en la región sinfisiaria.

El paciente no refiere ninguna sintomatología dolorosa y tampoco alteración de la sensibilidad en la zona afectada. No presenta en su historial médico ni odontológico ningún dato relevante y no tiene antecedentes de traumatismos. La lesión se descubre por hallazgo radiográfico 1 año después de la instalación de la aparatología ortodóncica.

En el examen extraoral no se observa asimetría facial. Al examen intraoral de la zona afectada, el vestíbulo se encontraba desocupado, sin abombamiento de las tablas óseas, los dientes involucrados tenían vitalidad pulpar positiva y una mucosa de características normales al tejido adyacente.

En los exámenes de imágenes, se le pidieron al ortodoncista las radiografías panorámicas de 1 mes antes de iniciar el tratamiento de ortodoncia y 1 año después de la instalación de la aparatología ortodóncica, en la cual se detectó que antes de iniciar el tratamiento ortodóncico, el paciente ya tenía la lesión. Las radiografías revelaron una imagen radiolúcida circunscrita, rodeadas por un halo radiopaco, de bordes irregulares, presentándose desde el incisivo lateral inferior (3.2) hasta el canino inferior (4.3), que se extendía festoneando las raíces de los dientes adyacentes (Fig. 1a y b). En la tomografía computarizada (TAC) se observó claramente la alteración de la arquitectura ósea, sin expansión de corticales, así como un adelgazamiento de las corticales lingual y vestibular (Fig. 2a y b) (Fig. 3a y b).

Se realizó una punción aspiración con aguja fina para descartar lesión quística, hemorrágica y sólida, de la que se obtuvo contenido líquido sanguinolento (Fig. 4).

Una vez completado el examen clínico y el análisis de los exámenes imagenológicos, se determina como hipótesis de diagnóstico clínico: quiste óseo simple.

Bajo anestesia local, se procedió a la exploración clínica del área afectada (Fig. 5a y b). La cavidad ósea estaba vacía y desprovista de tejido de revestimiento. A partir de esta observación, se confirmó el 


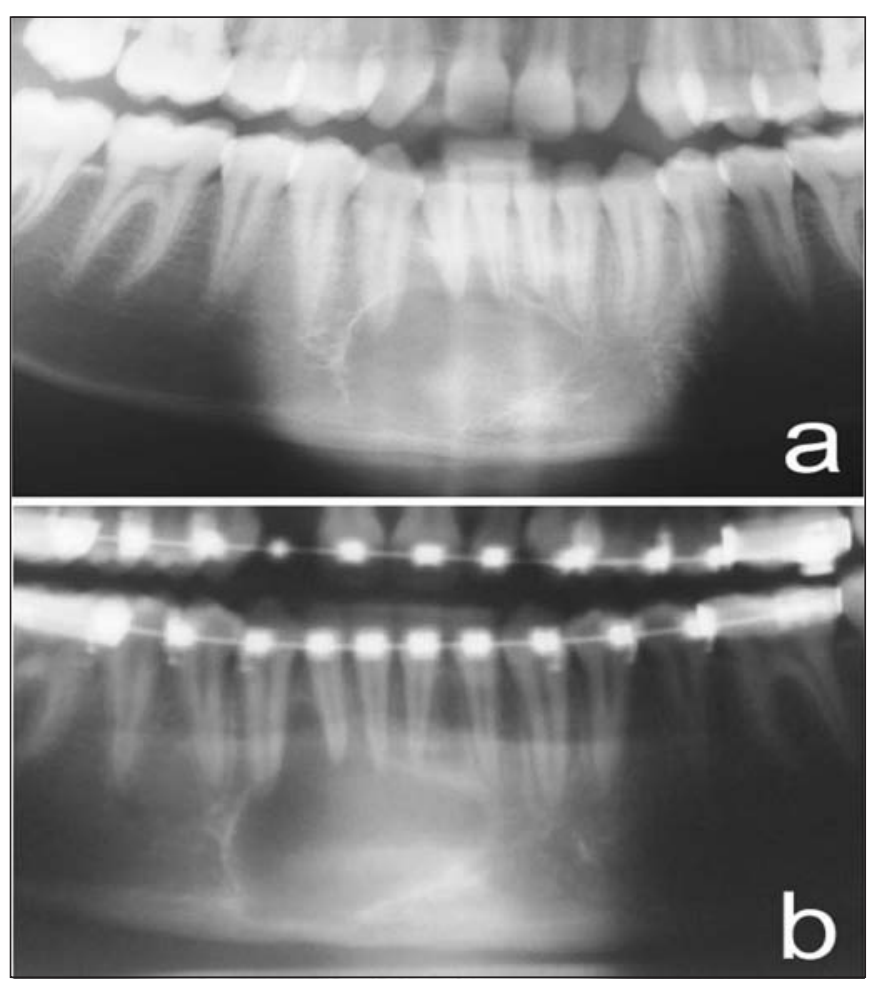

Fig. 1. a) Radiografía panorámica, tomada 1 mes antes de iniciar el tratamiento de ortodoncia. Nótese la presencia de una imagen radiolúcida que compromete la región sinfisiaria. b) Radiografía panorámica, tomada 1 año después instalación de la aparatología ortodóncica, se observa un aumento de la imagen radiolúcida que festonea las raíces de los dientes adyacentes.
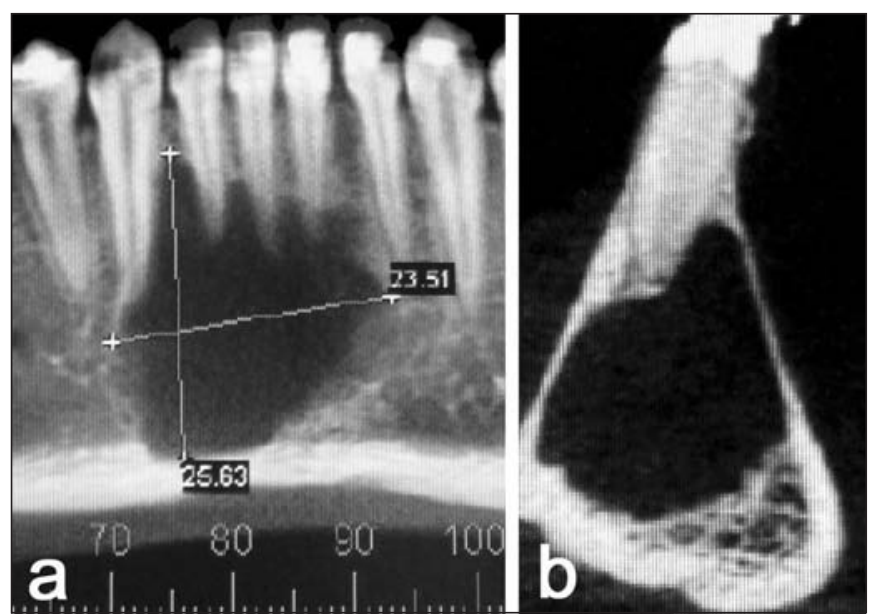

Fig. 2. a y b) Tomografía computarizada. Imagen panorámica y corte transversal, revelando la extensión de la lesión.

diagnóstico de quiste óseo simple. Se realizó legrado del lecho óseo con la finalidad de producir un sangrado en la cavidad y formación de coágulos de
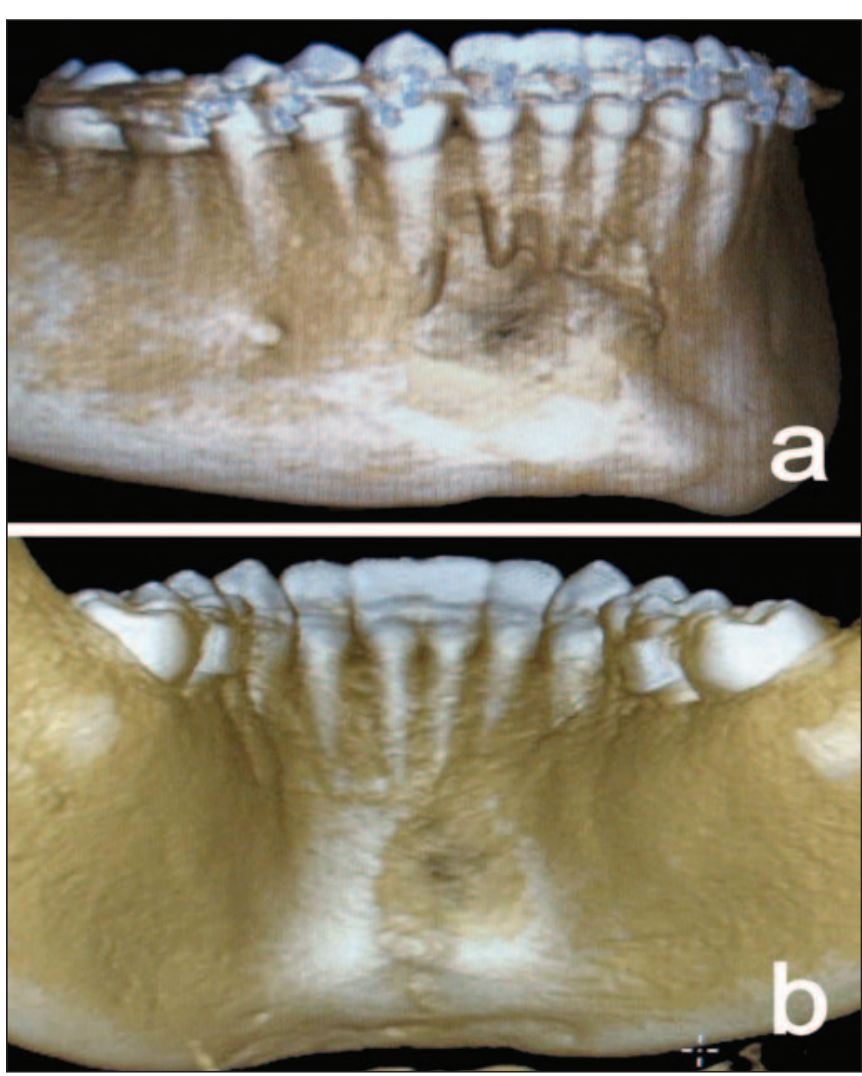

Fig. 3. a y b) Tomografía computarizada; las imágenes 3D demuestra alteración de la arquitectura ósea en la zona de sínfisis mandibular.

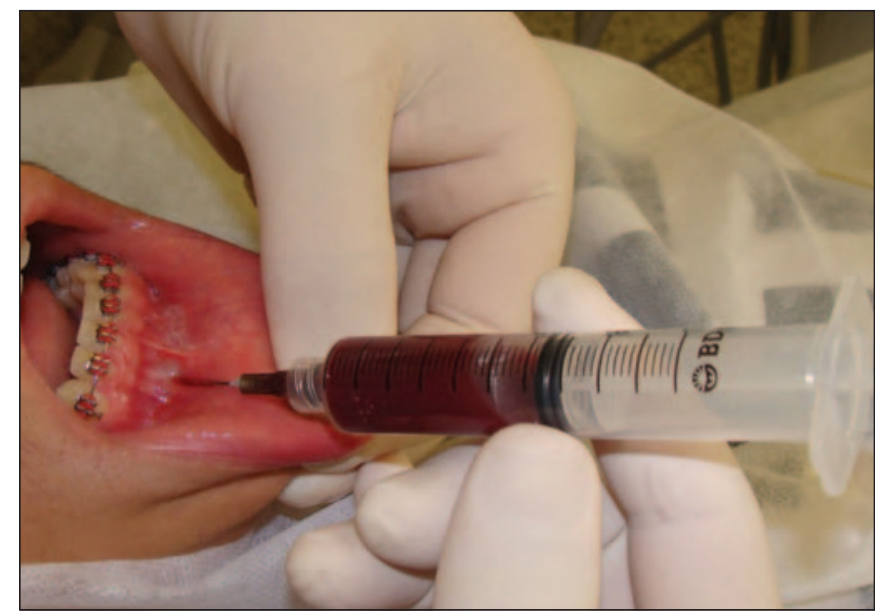

Fig. 4. Punción aspiración de la lesión (líquido sanguinolento).

sangre. El legrado no se hizo en la parte superior de la cavidad, para evitar la desvitalización de las piezas dentarias. 


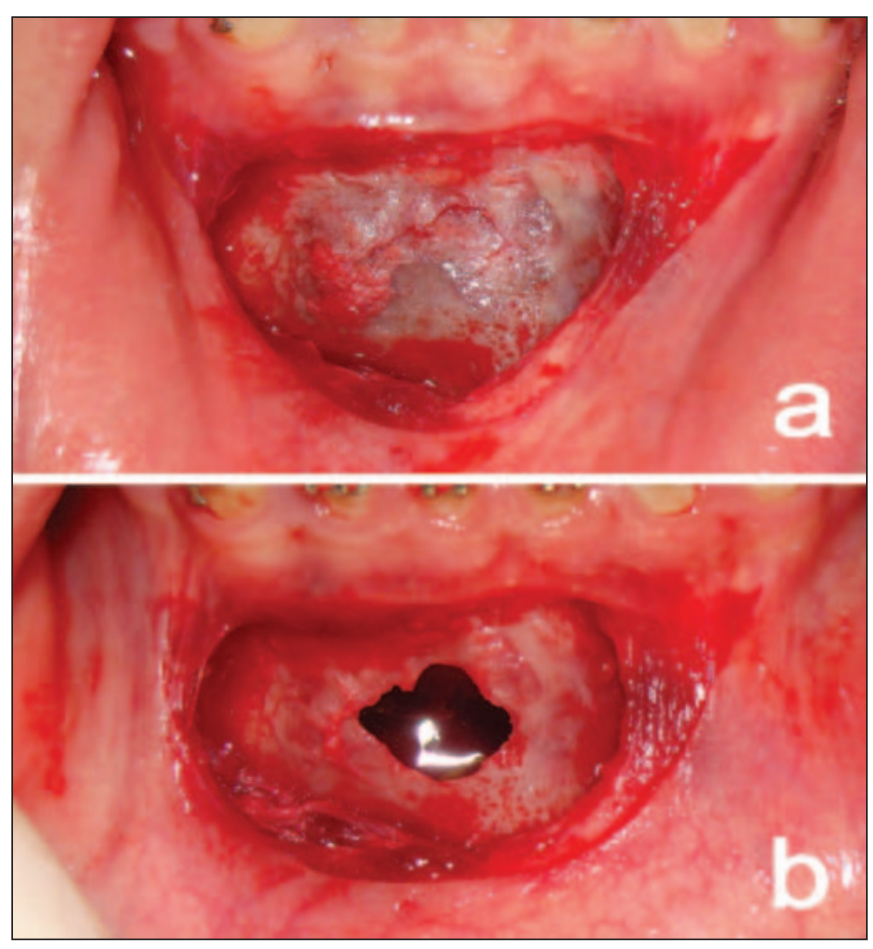

Fig. 5. a) Imagen intraoperatorio, donde se observa adelgazamiento de la cortical externa. b) Aspectos de la cavidad ósea, tras la apertura.

El postoperatorio transcurrió sin incidentes y a los 17 meses de seguimiento, la tomografía computarizada (TAC) mostró una restauración de la estructura ósea (Fig. 6a y b). Todos los dientes implicados en la lesión demostraron signos positivos en las pruebas de vitalidad pulpar.

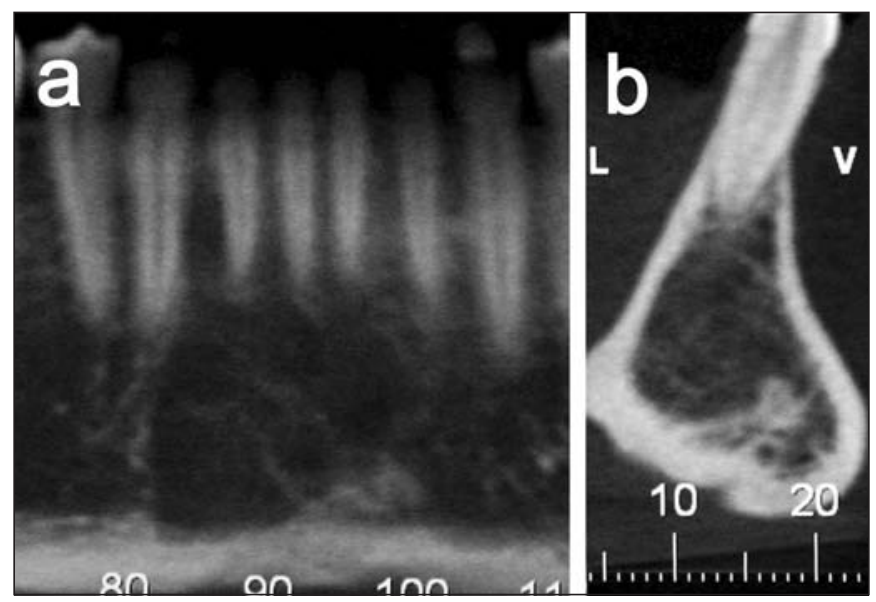

Fig. 6. a y b) Tomografía computarizada. Imágenes postoperatoria 17 meses después de la intervención mostrando una regeneración ósea en el área afectada.

\section{DISCUSIÓN}

En 1879, Virchow publicó el primer informe sobre el quiste óseo simple en el húmero. El extremo proximal del fémur, húmero y tibia son los lugares comunes de esta lesión (3, 11). En 1929, Lucas y Blum por primera vez describieron la lesión en la mandíbula (12). Sin embargo, no fue hasta 1946 que se establecieron los criterios de diagnóstico del quiste; que comprenden una lesión generalmente única sin recubrimiento epitelial, rodeada por paredes óseas, con un contenido liquido que en algunas ocasiones puede estar ausente y sin evidencia de infección (1).

En la clasificación más reciente de la Organización Mundial de la Salud (OMS), el QOS está incluido en el grupo de las "lesiones óseas no neoplásicas" junto a otras entidades como el quiste óseo aneurismático, fibroma osificante, displasia fibrosa, displasia óseo, granuloma central de células gigantes y querubinismo (13).

El QOS, ha recibido diversas denominaciones, dentro de las cuales se encuentran: quiste óseo traumático, quiste óseo solitario, cavidad ósea progresiva, cavidad ósea idiopática, quiste óseo unicameral, quiste de extravasación y quiste óseo hemorrágico $(8,14)$.

La etiología y patogénesis del QOS es desconocida, pero varias teorías se han propuesto, tales como:

1. Degeneración de los tumores óseos.

2. Alteraciones en el metabolismo de calcio.

3. Alteración local en el crecimiento óseo.

4. Aumento de la osteólisis.

5. Obstrucción venosa.

6. La hemorragia intramedular.

7. Leves cuadros infecciosos.

8. Isquemia local, o una combinación de esos factores $(15,16)$.

Diversos autores han descrito que un hecho traumático precedente, incluyendo una extracción dental puede dar lugar a un quiste de esta clase (14).

Varios autores registraron distintos porcentajes de pacientes con QOS con antecedentes de traumatis- 
mo orofacial: 23,8\%, Cortell-Ballester et al. (6), 2009; $28 \%$, Copete, et al. (17), 1998); y 71,4\%, PeñarrochaDiago et al. (8), 2001. El mecanismo más aceptado implica traumatismos que se traducen en una resolución o lisis defectuosa de la hemorragia intramedular que acaba con la formación de una cavidad ósea vacía $(14,18)$.

Los QOS suelen ser asintomáticos y en la mayoría de los casos son diagnosticadas por casualidad durante una exploración en un examen radiológico de rutina $(6,8)$. Las piezas dentarias que se localizan en estrecha relación con la cavidad intraósea, suelen conservarse vitales. En pocos casos se encuentra expansión cortical, fístula, parestesias, sensibilidad dental, dolor, linfadenopatía y fractura patológica (3, $7,15,17,19-21)$.

El diagnóstico del QOS está frecuentemente determinado por la asociación entre el examen clínico, los hallazgos radiográficos, la exploración quirúrgica, y el análisis histopatológico (cuando es posible) (22).

El diagnóstico diferencial descrito en la literatura incluye quiste periodontal lateral, periodontitis apical, quiste primordial, lesiones fibroóseas, quiste dentígero, queratoquiste, tumor odontogénico adenomatoide, ameloblastoma, granuloma central de células gigantes y fibroma ameloblástico $(22,23)$. Las dos últimas lesiones son especialmente importantes de destacar, ya que también suelen darse en el mismo grupo de edad y en lugares similares como ocurre con el QOS (17).

Radiológicamente el QOS se presenta como un área radiolúcida. Se manifiesta en la mayoría de las veces como una lesión unilocular aunque es posible encontrar multilocular, bien delimitada y con borde irregular $(6,9,24)$. El tamaño es variable y a veces puede presentarse con un fino reborde esclerótico ( 9 , 24). Cuando la lesión afecta a los espacios interdentarios, puede manifestar un aspecto lobular o festoneado. No suelen presentar expansión cortical. El desplazamiento dentario y la reabsorción de la raíz son poco frecuentes, y puede causar la desaparición de la lámina dura en el $14-83 \%$ de los casos $(13,25)$. En el caso presentado los hallazgos radiográficos fueron compatibles con los descritos en la literatura.
El examen histopatológico del QOS es muy variable y en algunos casos es posible identificar un delgado tejido conjuntivo laxo o fibroso bien vascularizado, sin evidencia de un componente epitelial, focos de células gigantes multinucleadas, tejido de granulación, zonas de hemorragia y focos de lesión fibroósea benigna. En cualquier caso, la ausencia de un revestimiento epitelial es una característica constante de estas lesiones $(3,6)$. Teniendo en cuenta los hallazgos clínicos y quirúrgicos de este caso se optó por no hacer el examen histopatológico.

El método más aceptado para el tratamiento del QOS es la exploración quirúrgica seguida de curetaje óseo de las paredes, con el objetivo de estimular la hemorragia para la formación de coágulos de sangre y que en la mayoría los casos dará lugar a una regeneración del tejido óseo $(6,9,10)$. No está indicado realizar tratamiento endodóntico en los dientes implicados, puesto que la pulpa dentaria se mantiene vital, incluso tras la intervención quirúrgica. Una encuesta de la literatura realizada por Huebner y Turlington en el año de 1971 demostró que el tiempo medio desde la cirugía hasta la curación completa era de 12,3 meses (26). Del mismo modo, en el estudio de Suei et al. en el año de 2007, la recuperación fue confirmado con mayor frecuencia entre 12 y 17 meses después de la cirugía (27). El protocolo utilizado para el tratamiento de este caso particular fue la exploración clínica y curetaje de la pared ósea mediante un acceso intraoral.

Algunos autores han señalado que el QOS puede resolver espontáneamente. Estos autores han reportado algunos casos, los cuales habían sido curados sin intervención quirúrgica (16). Sin embargo, la imposibilidad de exploración quirúrgica y examen histopatológico, puede dar lugar a la posibilidad de un error de diagnóstico. Por otra parte, al no realizarse un tratamiento puede dar lugar a complicaciones adicionales, tales como fractura mandibular patológica. Se han informado tres casos con una fractura patológica de la mandíbula se han reportado $(19,28,29)$.

Otros tratamientos alternativos consisten en el relleno de la cavidad con hueso liofilizado de la especie bovina o la introducción de sangre autóloga con el hueso del paciente o de hidroxiapatita. Estos trata- 
mientos pueden ser de interés en los casos en que falla el tratamiento convencional (30).

El pronóstico del quiste óseo simple es excelente y la tasa de recurrencia es baja $(9,20)$.

\section{CONCLUSIONES}

La revisión de la literatura y el caso clínico descrito permiten realizar las siguientes consideraciones:

- Los autores son bastantes uniformes con respecto a las características clínicas de los quistes óseos simples, relatando una mayor frecuencia durante la segunda década de la vida, asintomático, con una localización más frecuentemente en el cuerpo mandibular y en la mayoría de las veces los dientes de la zona presentan vitalidad pulpar.

- No todas las lesiones de quiste óseo simple presentan una alta probabilidad de un diagnóstico y por lo tanto, parece ser más prudente seguir utilizando la exploración quirúrgica y la evaluación histológica como la rutina de los medios para establecer el diagnóstico definitivo.

- La exploración quirúrgica seguida del curetaje de la lesión favorece la regeneración ósea progresiva. Este método ofrece un buen pronóstico; otras opciones de tratamiento sólo estarían justificadas en caso de recidiva.

- En la mayoría de los casos, la curación o la reaparición será confirmada después de los 3 años de la cirugía.

- La importancia de observar que no hayan alteraciones óseas y dentales antes de iniciar un tratamiento ortodóncico que indiquen la necesidad de un tratamiento adicional, o la intervención de otros tipos de especialistas antes de iniciar el tratamiento, como es el caso presentado donde el paciente inició el tratamiento con la lesión.

\section{BIBLIOGRAFÍA}

1. Rushton MA. Solitary bone cysts in the mandible. Br Dent J 1946;81(1):37-49.

2. Harris E, O’Carrol MK, Gandy F. Idiopatic bone cavity (Traumatic Bone Cyst) With the radiographic of a fibro-osseous lesion. Oral Surg. Oral Med. Oral Pathol 1992;74:118-23.

3. Saito Y, Hoshina Y, Nagamine T, Nakajima T, Suzuki M, Hayashi T. Simple bone cyst. A clinical and histopathologic study of fifteen cases. Oral Surg Oral Med Oral Pathol 1992;74: 487-91.

4. Freedman GL, Beigleman MB. The traumatic bone cyst: a new dimension. Oral Surg Oral Med Oral Pathol 1985;59:616-8.

5. Tomasi D, Hann JR. Traumatic bone cyst: report of case. J Am Dent Assoc 1985;111:56-7.

6. Cortell-Ballester I, Figueiredo R, Berini-Aytés L, GayEscoda C. Traumatic bone cyst: A retrospective study of 21 cases. Med Oral Patol Oral Cir Bucal 2009;14:239-43.

7. Gupta M, Kaste SC, Hopkins KP. Radiologic appearance of primary jaw lesions in children. Pediatr Radiol 2002;32:153-68.

8. Peñarrocha-Diago M, Sanchis-Bielsa JM, BonetMarco J, Minguez-Sanz JM. Surgical treatment and follow-up of solitary bone cyst of the mandible: a report of seven cases. Br J Oral Maxillofac Surg 2001;39:221-3.

9. Misino J, Lavis JF,Tardif A, Peron JM. Kystes solitaires des maxillares: traitement chirurgical et suivi de six case. Rev Stomatol Chir Maxillofac 2004;105:317-21.

10. Pradel W, Eckelt U, Lauer G. Bone regeneration after enucleation of mandibular cysts: comparing autogenous grafts from tissueengineered bone and iliac bone. Oral Surg Oral Med Oral Pathol Oral Rad and End 2006;101:285-90.

11. Shigematsu H, Fujita K, Watanabe K. Atypical simple bone cyst of the mandible. A case report. Int J Oral Maxillofac Surg 1994;23:298-9.

12. Lucas CD, Blum T. Do all cysts in the jaws originate from the dental system? J Am Dent Assoc 1929;16:647-61. 
13. Barnes L, Eveson JW, Reichart P, Sidransky D, editors. World Health Organization Classification of Tumours. Pathology and Genetics of Head and Neck Tumours. IARC Press: Lyon; 2005.

14. Pogrel MA. A solitary bone cyst possibly caused by removal of an impacted third molar. J Oral Maxillofac Surg 1987;45:721-3.

15. Cohen J. Etiology of simple bone cyst. J Bone Joint Surg 1970;52A:1493-7.

16. Sapp JP, Stark ML. Self-healing traumatic bone cysts. Oral Surg Oral Med Oral Pathol 1990;69: 597-602.

17. Copete MA, Kawamata A, Langlais RP. Solitary bone cyst of the jaws: radiographic review of 44 cases. Oral Surg Oral Med Oral Pathol Oral Radiol Endod 1998;85:221-5.

18. Sapp JP, Eversole LR, Wysocki GW. Lesiones óseas. En: Patología oral y maxilofacial contemporánea. $2^{a}$ ed. Madrid .Elsevier, 2005: 123-4.

19. Baird WO, Askew PA. Traumatic mandibular bone cyst involved in line of fracture. Oral Surg Oral Med Oral Pathol 1958;11:1351-6.

20. Baqain ZH, Jayakrishnan A, Farthing PM, Hardee P. Recurrence of a solitary bone cyst of the mandible: case report. Br J Oral Maxillofac Surg 2005;43:333-5.

21. Eriksson L, Hansson GL, Åkesson L, Ståhlberg F. Simple bone cyst: a discrepancy between magnetic resonance imaging and surgical observations. Oral Surg Oral Med Oral Pathol Oral Radiol Endod 2001;92:694-8.

22. Rodrigues CD, Estrela C. Traumatic bone cyst suggestive of large apical periodontitis. J Endod 2008;34:484-9.

23. Rosen DJ, Ardekian L, Machtei EE, Peled M, Manor R, Laufer D. Traumatic bone cyst resembling apical periodontitis. J Periodontol 1997;68:1019-21.

24. Matsuzaki H, Asaumi JI, Yanagi Y, Konouchi $H$. Honda Y, Hisatomi M, Shigehara H, Kishi K. MR imaging in the assessment of a solitary bone cyst. Eur J Radiol Extra 2003;45:37-42.

25. Davis WM Jr, Buchs AU, Davis WM. Extravasation cyst diagnostic curettement: a treatment. Report of 15 cases and suggested treatment. Oral Surg Oral Med Oral Pathol. 1979;47:2-7.

26. Huebner GR, Turlington EG. So-called traumatic (hemorrhagic) bone cysts of the jaws. Review of the literature and report of two unusual cases. Oral Surg Oral Med Oral Pathol. 1971;31:354-65.

27. Suei Y, Taguchi A, Tanimoto K. Simple bone cyst of the jaws: evaluation of treatment outcome by review of 132 cases. J Oral Maxillofac Surg. 2007; 65:918-23.

28. Hughes CL. Hemorrhagic bone cyst and pathologic fracture of mandible: report of case. J Oral Surg 1969;27:345-6.

29. Matise JL, Beto LM, Fantasia JE, Fielding AF. Pathologic fracture of the mandible associated with simultaneous occurrence of an odontogenic keratocyst and traumatic bone cyst. J Oral Maxillofac Surg. 1987;45:69-71.

30. Dellinger TM, Holder R, Livingston HM, Hill WJ. Alternative treatments for a traumatic bone cyst: a longitudinal case report. Quintessence Int. 1998;29:497-502.

\section{CORRESPONDENCIA}

Rodrigo López Alvarenga

Gomera, 6, esc. 2, $3^{\circ} \mathrm{H}$

04770 Adra. Almería

E-mail:mre_lopez@hotmail.com 